\title{
Propostas de Técnicas de Coexistência entre LTE-U e Wi-Fi em 5 GHz considerando diferentes modulações candidatas $5 \mathrm{G}$
}

\author{
Pedro Manoel Ribeiro Rosa ${ }^{1}$, Maykon Renan Pereira da Silva ${ }^{2}$, \\ Flávio Geraldo Coelho Rocha ${ }^{2}$, Vinicius da Cunha Martins Borges ${ }^{1}$ \\ ${ }^{1}$ Instituto de Informática - Universidade Federal de Goiás (UFG) \\ Alameda Palmeiras, Quadra D, Câmpus Samambaia - Goiânia-GO \\ ${ }^{2}$ Escola de Engenharia Elétrica, Mecânica e de Computação \\ Universidade Federal de Goiás (UFG) \\ Av. Universitária, n. ${ }^{\circ}$ 1488, Qd. 86, Setor Leste Universitário - Goiânia-GO \\ \{vinicius, flaviogcr\}@ufg.br, \{pedromanoel, maykonrenan\}@discente.ufg.br
}

\begin{abstract}
The growing demand for mobile LTE connections has resulted in the saturation of the available spectrum, motivating operators to search new frequency spectral such as the $5 \mathrm{GHz}$ band as it is not licensed and little used and having the IEEE $802.11 \mathrm{a} / \mathrm{n} / \mathrm{ac}$ standards of Wi-Fi operating on that same frequency. This article proposes the LAS and RABS coexistence techniques applied in $5 G$ candidate modulations (F-OFDM/UFMC) allowing LTE- $U$ and Wi-Fi to operate simultaneously, achieving better performance for Wi-Fi traffic when compared to the technique ABS.
\end{abstract}

Resumo. A crescente demanda de conexões móveis do LTE tem como consequência a saturação do espectro disponível, motivando as operadoras a buscarem novos espectros de frequência como a faixa de $5 \mathrm{GHz}$ por não ser licenciada e pouco utilizada e tendo os padrões IEEE 802.1 la/n/ac do Wi-Fi operando nessa mesma frequência. Esse artigo propõe as técnicas de coexistência LAS e RABS aplicadas nas modulações candidatas à $5 G$ (F-OFDM/UFMC) permitindo o LTE-U e Wi-Fi operarem de modo simultâneo, conseguindo melhor desempenho ao tráfego Wi-Fi quando comparadas com a técnica ABS.

\section{Introdução}

$\mathrm{O}$ aumento em número e diversidade de equipamentos conectados nas redes de dados sem fio de $3^{\mathrm{a}}$ e $4^{\mathrm{a}}$ Geração, $3 \mathrm{G}$ e $4 \mathrm{G}$, é uma realidade conforme o documento Cisco Visual Networking Index: Global Mobile Data Traffic Forecast Update, 2018-2023, publicado em março de 2020 por [CISCO 2020], dois terços da população mundial terão acesso à Internet até 2023. Isso significa um total de 5 bilhões e 300 milhões de usuários conectados à Internet, sendo um crescimento de $51 \%$ comparado com o ano de 2018 , representando um aumento de 3 bilhões e 900 milhões de novos usuários conectados à Internet.

Nesse cenário de crescimento de tráfego, existe uma série de fatores que limita a expansão dessas conexões de acesso sem fio, dentre elas a escassez do espectro rádio disponível e os efeitos de propagação de sinal são desafiadores, pois limitam a eficiência da rede. A tendência de topologia entre as operadoras é a troca de tráfego de macro células para micro células, permitindo-lhes redução de custo e aumento da capacidade. 
Vale mencionar os dados sobre o uso das redes móveis por meio da tecnologia Wireless Fidelity - Wi-Fi e da rede celular, padrão Long Term Evolution - LTE, por um mesmo dispositivo. Muitas dessas conexões móveis ocorrem nas residências dos usuários com banda larga fixa ou pontos de acesso Wi-Fi ou, ainda, são atendidos por femtocell ou picocell das operadoras de celular. Uma considerável proporção de tráfego gerado por esses dispositivos é descarregada da rede móvel para a rede fixa, caracterizando essa operação por dual-mode.

Essa escassez de espectro pode ser evidenciada pelo modo de operação de $5 \mathrm{G}$ das operadoras VIVO, TIM e CLARO, que operam no BRASIL. Conforme [Bonatelli 2020], essas operadoras irão compartilhar o espectro licenciado da $4 \mathrm{G}$ com a $5 \mathrm{G}$, por meio do mecanismo Dynamic Spectrum Sharing - DSS, até ocorrer o leilão das frequências destinadas a 5G, previsto para o ano de 2021 com data a ser definida.

Apesar de existirem trabalhos que propõem o uso das ondas milimétricas pelo LTE na faixa de 30 ou 60 Giga Hertz - GHz, em espectro não licenciado, há desvantagens em sua utilização como atenuações pelo desvanecimento de percurso, aumento da absorção atmosférica pela umidade, neblina ou chuva, perdas de penetração e reflexão. Além disso, as transmissões utilizando ondas milimétricas não são adequadas para transmissões setoriais, pois o comprimento de onda mais curto proporciona o aumento significativo da suscetibilidade ao bloqueio pelos obstáculos, conforme [Ichkov et al. 2017].

Por outro lado, trabalhos como [Gerzaguet et al. 2017], [Liu et al. 2016], [Lizeaga et al. 2017] apresentam um estudo comparativo das modulações Filtered Orthogonal Frequency Division Multiplexing - F-OFDM e Universal Frequency Multicarrier - UFMC candidatas à 5G para a telefonia móvel, trazendo em seu corpo definições e parâmetros utilizados a fim de obter melhor qualidade de comunicação e eficiência no uso do espectro em relação à modulação Orthogonal Frequency Division Multiplexing OFDM. Nesse aspecto de eficiência, as modulações candidatas apresentaram consumo menor de energia na formação do sinal, proporcionando menor emissão fora da banda facilitando a coexistência.

As modulações candidatas lidam com a interferência na coexistência entre WiFi e LTE-U de forma diferente das técnicas de coexistência, explorando outros aspectos. Logo, as modulações e técnicas poderiam trabalhar de forma complementar para melhorar a eficiência do tráfego Wi-Fi. Desta forma, uma combinação das modulações candidatas com as técnicas de coexistência existentes faz-se útil para investigar o impacto dessa combinação na coexistência dessas duas tecnologias de transmissão da camada física, em ambas perspectivas, tanto do Wi-Fi quanto LTE-U.

Sendo assim, como solução eventual da escassez de espectro licenciado, as técnicas de coexistência permitem ao LTE operar juntamente com o Wi-Fi no espectro não licenciado de $5 \mathrm{GHz}$. Vale mencionar que o uso do espectro não licenciado pelo LTE em caráter eventual/secundário é chamado de Long Term Evolution-Unlicensed - LTE-U.

Portanto, o objetivo principal desse trabalho é propor duas técnicas de coexistência Low Amplitude Signal — LAS e Reduce Amplitude with Blank Subframes - RABS decorrentes das técnicas de coexistência Almost Blank Subframes - ABS e Low Amplitude Stream Injection - LASI, permitindo o LTE-U e Wi-Fi operarem de modo simultâneo e sem degradação da potência de transmissão do Wi-Fi. 
Nesse artigo a seção 2 mostra os trabalhos relacionados sobre as técnicas de coexistência, enquanto que a seção 3 expõe as considerações sobre as técnicas de coexistência. A seção 4 apresenta as propostas das técnicas de coexistência LAS e RABS. A seção 5 mostra o cenário das simulações e avaliação enquanto que a seção 6 a conclusão e os trabalhos futuros.

\section{Trabalhos Relacionados}

O estudo sobre técnicas de coexistência em espectro não licenciado tem sido alvo de pesquisa desde 2014, como se observa nas Releases 13 e 14 do 3rd Generation Partnership Project - 3GPP e, atualmente, pelos trabalhos da Qualcomm [FCC 2016b], Nokia [FCC 2018] e MulteFire Alliance [MULTEFIRE 2018]. Importante mencionar que as técnicas de coexistência ABS e Listen Before Talking - LBT foram propostas nas Releases 10 [3GPP 2009] e 13 [3GPP 2016] do 3GPP, respectivamente, enquanto que a Carrier-Sensing Adaptive Transmission - CSAT foi introduzida pela Qualcomm [QUALCOMM 2014].

Cui et al. [Cui et al. 2017] e Huang et al. [Huang et al. 2018] descrevem as técnicas de coexistência LBT, ABS e CSAT, considerando a coexistência entre LTE-U e Wi-Fi. Ambos explicam que a técnica ABS permite o Wi-Fi acessar o canal livre de interferência durante uma pequena "janela" de tempo proporcionada pelos subframes em branco. Com relação ao CSAT empregado no LTE-U, o autor descreve como sendo uma técnica de coexistência que analisa a duração média que o Wi-Fi ocupa o canal. Com base nesse período o LTE-U poderá ocupar o canal, pois consegue identificar o período em que o Wi-Fi não estará utilizando-o.

Sun et al. [Sun et al. 2017] apresentam a técnica LASI que consiste em diminuir a amplitude do sinal do Wi-Fi, consideravelmente. Ainda, propõe o uso do algoritmo Conflict-Tolerant Channel Allocation - CTCA em conjunto com a LASI, para auxiliar na recuperação da informação perdida pelo Wi-Fi. É necessário em sua aplicação a coordenação entre o LTE-U e Wi-Fi.

Abinader et al. [Abinader et al. 2018] explicam a coexistência das tecnologias LTE-U e Wi-Fi, operando em $5 \mathrm{GHz}$, ou seja, na banda de uso da indústria, ciência e saúde. Propõe a técnica de coexistência LTE Blank Subframes, bastante similar à técnica ABS diferindo-se pela alocação dinâmica da quantidade de subframes em branco, podendo alocar quantidades diferentes em uma transmissão LTE-U com base na performance do tráfego Wi-Fi.

Bocanegra et al. [Bocanegra et al. 2019] implementaram o LTE-U coexistindo com o Wi-Fi 802.11ac utilizando a técnica de coexistência ABS. Ainda, o autor propõe um algoritmo em que o nó mais distante possa realizar uma transmissão direta ao nó mais próximo, tornando-o um retransmissor do sinal. Vale mencionar que o autor configurou em $20 \mathrm{MHz}$ a largura de banda para o IEEE 802.11ac do Wi-Fi.

\section{Considerações Sobre as Técnicas de Coexistência}

As técnicas LBT, Fair-LBT, ALOHA-Like e CSAT trabalham similarmente ao mecanismo Carrier Sense Multiple Access with Collision Avoidance - CSMA-CA do padrão Wi-Fi, não considerando o uso simultâneo do canal pelo LTE-U e Wi-Fi. Essas técnicas 
podem prejudicar o tráfego Wi-Fi em cenário densos, uma vez que o LTE-U tendo ocupado o canal livre e com muito tráfego o Wi-Fi poderá não conseguir ocupá-lo por meio do CSMA-CA e, por conseguinte, reiniciar todo o processo de acesso ao meio.

A técnica LASI consiste na diminuição de potência do sinal Wi-Fi quando ocorrer a disputa do canal, sendo empregada em conjunto com o algoritmo CTCA para recuperação de pacotes em conflito com o LTE-U. A diminuição da potência do sinal Wi-Fi torna-se um risco ao seu tráfego, uma vez que sua potência de $50 \mathrm{~mW}(17 \mathrm{dBm})$ é muito inferior à do LTE-U sendo mais suscetível à interferência. Outra desvantagem encontra-se na necessidade de coordenação entre a estação base e o ponto de acesso para que a diminuição de potência do sinal Wi-Fi possa ocorrer. Tal procedimento na prática torna-se inviável, devido os pontos de acesso não possuírem mecanismos embarcados para tal coordenação.

A técnica ABS permite a utilização do canal de forma simultânea para o LTE-U e Wi-Fi, não exigindo nenhuma "escuta" do canal e tampouco coordenação entre a estação base e o ponto de acesso. Ela se destaca entre as demais pela possibilidade de coexistência simultânea do canal independente de idles de transmissão do Wi-Fi devido os subframes em branco, como também por não precisar de coordenação com o Wi-Fi. Porém não se torna escalável à medida que o cenário torna-se denso por não oferecer maior número de subframes em branco.

Diante o exposto e considerando as vantagens e desvantagens de cada técnica de coexistência, tendo como pressuposto a operação simultânea entre o LTE-U e Wi-Fi, este trabalho propõe as técnicas de coexistência LAS e RABS combinadas com as modulações empregadas no LTE-U. Importante ressaltar que combinar essas técnicas de coexistência com as modulações candidatas à 5G (F-OFDM/UFMC) poderão favorecer tanto o tráfego LTE-U quanto Wi-Fi, uma vez que tais modulações emitem menor vazão fora da banda promovendo menor interferência entre os sinais adjacentes.

\section{Técnicas de Coexistência Propostas}

Nesta sessão serão apresentadas as técnicas propostas de coexistência LAS e RABS, descrevendo o funcionamento e os princípios adotados das técnicas de coexistências ABS e LBT utilizadas nos Releases 10 [3GPP 2009] e 13 [3GPP 2016] do 3GPP, respectivamente.

\subsection{Técnica de coexistência LAS (Low Amplitude Signal)}

A motivação da proposta da técnica LAS parte de duas desvantagens que a técnica LASI de Sun et al. [Sun et al. 2017] apresenta, quais sejam, coordenação entre a Base Station BS (LTE-U) e o Access Point - AP (Wi-Fi) que está na região de coexistência e diminuição da potência do AP. A coordenação entre a BS e o AP atualmente torna-se impraticável devido os APs residenciais não possuírem uma interface própria ou sinalização em sua transmissão para que ocorra essa troca de informações com a BS, além de não garantir a escalabilidade com uma grande quantidade de APs existentes nas small cells.

Nesse contexto, a Tabela 1 apresenta as principais diferenças entre a técnica proposta LAS e a LASI. A técnica proposta LAS, assim como a LASI, é aplicada no LTE-U e possui a vantagem de não exigir qualquer tipo de coordenação entre a BS e AP. A Fi- 
gura 1 apresenta o funcionamento básico da técnica proposta LAS, onde o sinal da BS é atenuado quando há a presença do sinal Wi-Fi.

Tabela 1. Comparação entre as Técnicas LASI e LAS, do autor.

\begin{tabular}{lcc}
\hline \multicolumn{1}{c}{ Característica } & LASI & LAS \\
\hline Aplicação/Implementação & LTE-U e Wi-Fi & LTE-U \\
Coordenação & Com o Wi-Fi & Não Precisa \\
Atenuação de Potência & No AP & Na BS \\
Esquema de Modulação & 256-QAM & Adaptável \\
\hline
\end{tabular}

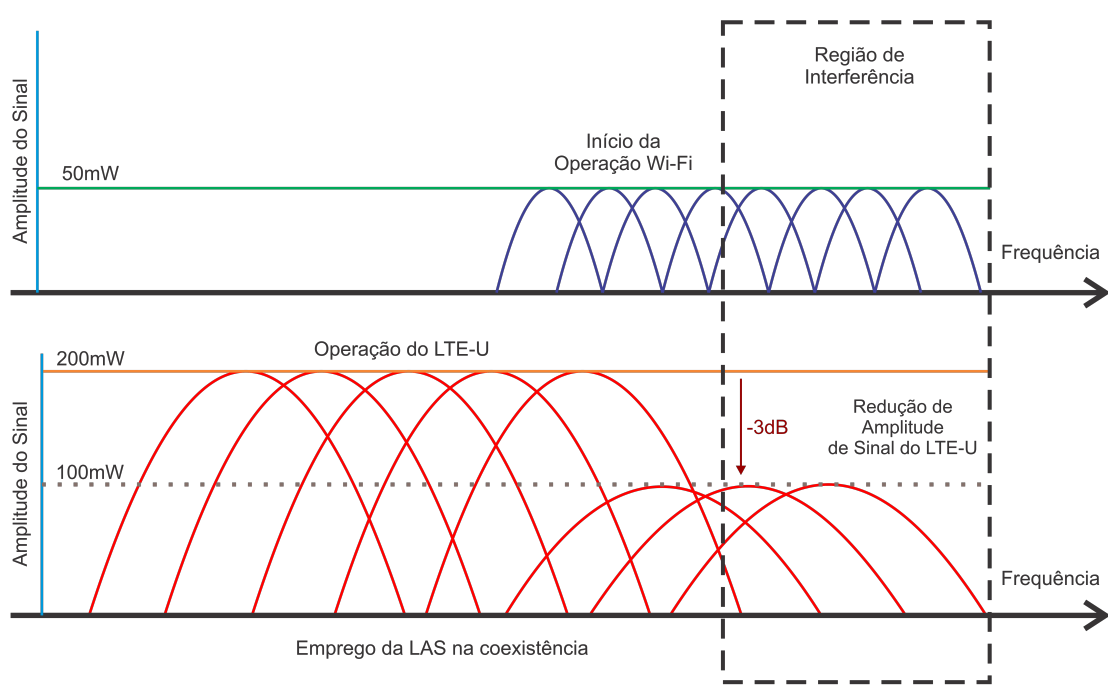

Figura 1. Operação do LTE-U e Wi-Fi c/ LAS, do autor.

A Figura 2 apresenta um estudo empírico sobre o impacto da redução de potência no sinal do LTE-U de forma a entender melhor qual poderia ser um valor de potência reduzido para um cenário real de coexistência.

A Tabela 2 apresenta os dados estratificados da Figura 2(a) com as vazões alcançadas à media que se atenua a potência do LTE-U. Nesse contexto, verifica-se uma queda da vazão de $35 \%$ com a potência de $50 \mathrm{~mW}(17 \mathrm{dBm})$ em relação à potência de 200 $\mathrm{mW}(23 \mathrm{dBm})$. Por outro lado, há uma queda de $8 \%$ com a potência de $100 \mathrm{~mW}(20 \mathrm{dBm})$ em relação à potência de $200 \mathrm{~mW}(23 \mathrm{dBm})$, dessa forma, torna-se possível atenuar em $3 \mathrm{~dB}$, pois a vazão com $23 \mathrm{dBm}$ se assemelha bastante com a vazão em $20 \mathrm{dBm}$ quando variando a Signal-to-Interference-plus-Noise Ratio - SINR.

Tabela 2. Vazões Médias da Figura 2(a).

\begin{tabular}{ccc}
\hline Potência LTE-U & Vazão Média (Mbps) & Relação Percentual \\
\hline $200 \mathrm{~mW}$ & $\mathbf{3 , 1 6}$ & $\mathbf{1 0 0 \%}$ \\
$100 \mathrm{~mW}$ & $\mathbf{2 , 8 8}$ & $\mathbf{9 2 \%}$ \\
$50 \mathrm{~mW}$ & $\mathbf{2 , 0 5}$ & $\mathbf{6 5 \%}$ \\
\hline
\end{tabular}


Vale mencionar que a potência máxima de transmissão em espectro não licenciado não poderá ultrapassar os $200 \mathrm{~mW}(23 \mathrm{dBm})$, preconizada pela Federal Communications Commission - FCC [FCC 2016a]. Embora até o presente momento não tenha sido empregada a atenuação de amplitude do sinal LTE-U por meio da redução de potência no emprego das técnicas de coexistência, tanto quanto sabemos torna-se a primeira abordagem que leva em consideração esse aspecto. Importante ressaltar que a potência de transmissão do LTE-U inicia com valor máximo permitido pela FCC, enquanto que a potência de transmissão do Wi-Fi se mantém constante em $50 \mathrm{~mW}(17 \mathrm{dBm})$. Ainda, a área de cobertura da small cell, tanto do LTE-U quanto Wi-Fi, é um fator que possibilita o uso de potência com os valores menores que o especificado pela FCC.

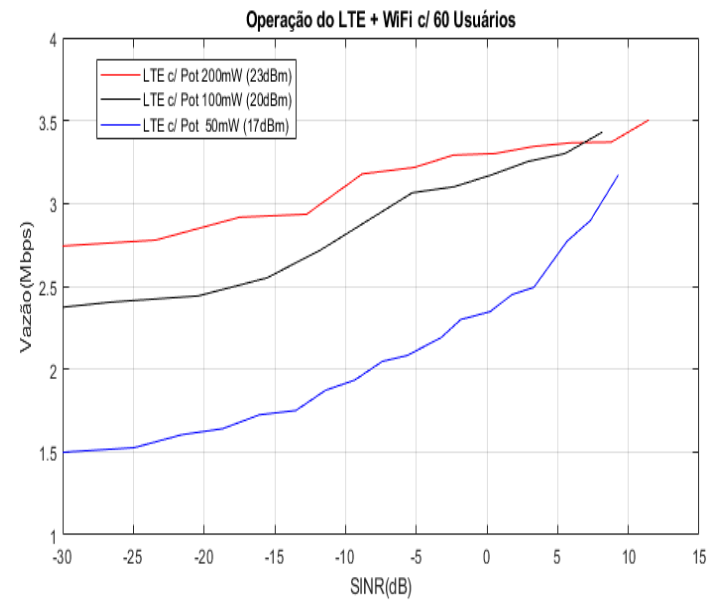

(a) Vazão c/ Variação de Potência do LTE-U

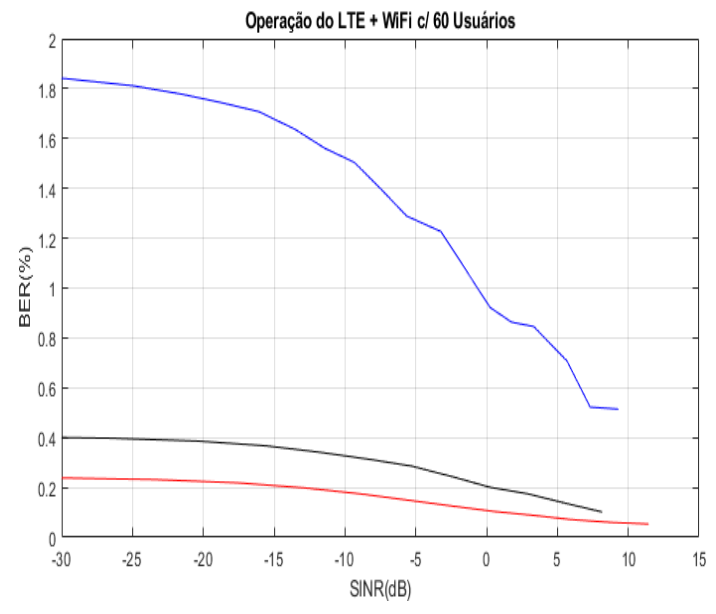

(b) BER c/ Variação de Potência do LTE-U

Figura 2. Desempenho do LTE-U c/ Variação da Potência, do autor.

Em complemento, a Figura 2(b) mostra o comportamento do BER em relação à variação de potência do LTE-U. Nesse contexto, verifica-se a maior perda de bits quando aplicada a potência de $50 \mathrm{~mW}$ nas transmissões do LTE-U, em relação à aplicação de potência de $100 \mathrm{~mW}$ ou $200 \mathrm{~mW}$. Vale considerar que essa simulação foi realizada no cenário mais denso, ou seja, com 60 usuários com um raio de $30 \mathrm{~m}$ de comprimento, onde é possível concluir que a redução de potência em $3 \mathrm{~dB}$ torna-se viável para os tráfegos do LTE-U e Wi-Fi.

Diante disso e levando em consideração o cenário de uma small cell, foi possível uma análise para adequar uma determinada potência ao LTE-U sem prejuízo significativo ao seu tráfego. Portanto, dependendo do número de usuários, da distância entre eles e suas respectivas fontes de transmissão, tanto do LTE-U quanto do Wi-Fi, a redução da potência proposta gerou um bom trade-off entre LTE-U e Wi-fi quando avaliando Bit Error RateBER, Block Error Rate - BLER e Vazão dessas duas tecnologias.

\subsection{Técnica de coexistência RABS (Reduce Amplitude with Blank Subframes)}

Nas pesquisas dos trabalhos apresentados sobre coexistência do LTE-U e Wi-Fi, as técnicas mais utilizadas como ABS e LBT são implementadas no LTE-U por considerar devido o uso do espectro licenciado de modo secundário/eventual como também pela robustez da formação do sinal e do seu mecanismo de recuperação da informação. Em 
decorrência, as técnicas de coexistência atenuam a eficiência de seu tráfego para favorecer o tráfego Wi-Fi.

Assim sendo, a aplicação da técnica LAS irá proporcionar menor interferência ao tráfego Wi-Fi devido a consequente atenuação da Out of Band Emission - OoBE das modulações que operam no LTE-U, levando em consideração que a potência de transmissão da BS está no limite permitido pela FCC [FCC 2016a] e sendo suficiente para abranger os usuários móveis situados nas small cells. Entretanto, a diminuição da potência de transmissão do LTE-U não irá oportunizar os slots de tempo necessários para que o mecanismo CSMA-CA do Wi-Fi possa estabelecer o melhor momento da transmissão do Wi-Fi e, ainda, contribuir para evitar as colisões.

Nesse sentido, para que o Wi-Fi possa executar o CSMA-CA com sucesso e, como consequência, otimizar suas transmissões em coexistência com o LTE-U, a proposta da técnica de coexistência RABS irá consistir na junção das técnicas de coexistência: LAS e ABS. Dessa forma, a técnica proposta RABS irá oportunizar slots de tempo para o WiFi por meio da inserção dos cinco subframes em branco no frame do LTE-U conforme a Figura 3. Logo em seguida, a técnica RABS irá atenuar a potência de transmissão do LTE-U de $200 \mathrm{~mW}(23 \mathrm{dBm})$ para $100 \mathrm{~mW}(20 \mathrm{dBm})$ a fim de diminuir a emissão fora da banda das modulações que operam no LTE-U. Isso possibilitaria melhor escalabilidade, portanto, sendo mais voltado para cenários densos.

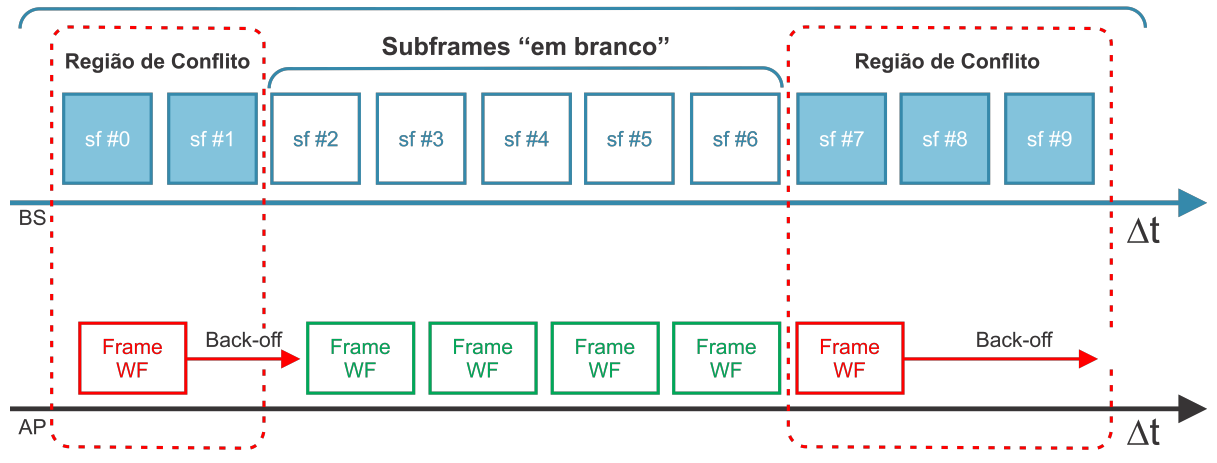

Figura 3. CSMA-CA e Téc. RABS, do autor.

Portanto, com a técnica proposta RABS o Wi-Fi transmite no canal como se estivesse "ocioso" através dos subframes em branco do frame do LTE-U e, ao mesmo tempo, sofre menor interferência resultante pela atenuação da emissão fora da banda da modulação empregada no LTE-U.

Nesse contexto, a técnica RABS atua no processo de Primary Download Shared Channel - PDSCH, onde os subframes em branco são definidos, logo após, diminui o valor da potência de transmissão do sinal pela metade (3dB). Com isso, torna-se possível diminuir os efeitos da interferência decorrentes do uso simultâneo do canal pelo LTE-U e Wi-Fi, proporcionando desempenho favorável para o tráfego do Wi-Fi.

\section{Cenário e Avaliação}

Nesta seção serão apresentadas as informações a respeito do cenário, configuração e implementação das simulações realizadas e parâmetros do LTE-U e Wi-Fi, bem como a análise dos resultados apresentados pelas modulações, combinadas ou não, com as técnicas de coexistências. 


\subsection{Descrições do Cenário e Simulações}

Nas simulações foram empregados os padrões LTE e o IEEE 802.11ac do Wi-Fi operando simultaneamente, sendo implementada toda estrutura da camada física do Evolved Universal Terrestrial Radio Access Network - E-UTRAN, de acordo com os Relases 10 e 13 do $3 \mathrm{GPP}$.

O cenário é retratado na Figura 4, em que temos a BS e o User Equipament - UE do lado do LTE, e o AP e o Nó do lado Wi-Fi. O deslocamento dos usuários acontece da extremidade para o centro ao passo de 1 metro, efetuando um deslocamento total de 30 metros. Da mesma forma, o UE também realiza esse deslocamento, iniciando-se da BS até o centro. Vale mencionar que a distância entre a BS e o AP é de 60 metros com 20, 40 e 60 usuários, sendo uma metade UE e a outra nós do Wi-Fi.

Para elaboração do cenário, a distâncias de $60 \mathrm{~m}$ e de deslocamento dos usuários foram baseados conforme [Bocanegra et al. 2019] e [Sun et al. 2017]. A área indoor e os parâmetros de canalização e desvanecimento do canal estão de acordo com [ITU 2012], tendo sido empregados também nos experimentos de [Bocanegra et al. 2019], [Sun et al. 2017] e [Tariq et al. 2018]. A quantidade de usuários foi dimensionada tomando-se como referência o número de usuários de uma small cell, previsto em [Beard and Stallings 2016]. Vale mencionar que Abinader et al. [Abinader et al. 2018] e Bocanegra et al. [Bocanegra et al. 2019] utilizaram quantidades de usuários similares ao cenário proposto neste trabalho.

O cenário da simulação foi construído sob os parâmetros da International Telecommunication Union - ITU [ITU 2012] para transmissões do tipo indoor. O código foi implementado na plataforma MATLAB ${ }^{\circledR}$, versão 2018 b, utilizando a LTE ToolBox e a WLAN System ToolBox.

A Tabela 3 traz as configurações do LTE-U utilizadas em todas as modulações candidatas (F-OFDM, UFMC e FBMC), incluindo a OFDM. Importante notar que foi adotado o esquema de modulação $Q u a-$ drature Phase Shift Keying - QPSK, tendo o Transport Block Size - TBS um valor de

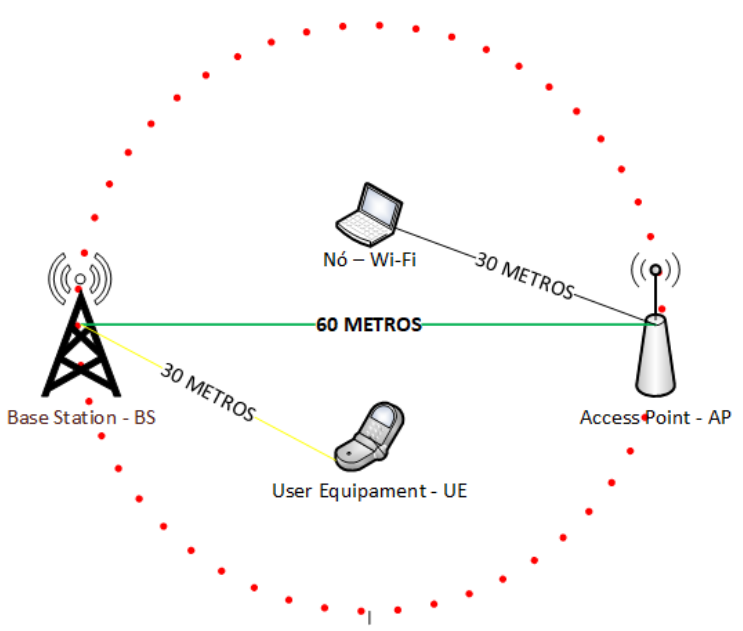

Figura 4. Cenário da Simulação, do autor. 10.296 bits $\left(I_{T B S}=6\right)^{1}$ para 100 blocos de recursos, uma vez que se considera o pior cenário nas simulações devido a presença da interfêrencia de sinal.

A Tabela 4 apresenta os parâmetros de configuração do Wi-Fi utilizados nas simulações deste trabalho. Ressalta-se o mesmo esquema de codificação empregado no padrão LTE-U, por considerar o pior cenário na coexistência. A Tabela 5 retrata as potências de transmissão da BS e do AP utilizadas inicialmente nas simulações, respeitando a recomendação de [FCC 2016a].

\footnotetext{
${ }^{1}$ Conforme a Tabela 7.1.7.2.1-1, da TS 36.213 da 3GPP, versão 15.2.0 Release 15, de abril de 2018.
} 
Tabela 3. Parâmetros do LTE-U

\begin{tabular}{ll}
\hline \multicolumn{2}{c}{ Parâmetros do LTE-U } \\
\hline Largura de Banda & $20 \mathrm{MHz}$ \\
Modo Duplex & FDD \\
Frequência de Operação & $5 \mathrm{GHz}$ \\
Indicador de Qualidade do Canal & $8 \mathrm{~ms}$ \\
Esquema de Modulação & QPSK \\
Ruído de Fundo & $-95 \mathrm{dBm}$ \\
$\mathrm{N}^{\circ}$ de Blocos de Recursos & 100 \\
$\mathrm{~N}^{o}$ de Elementos de Recursos & 12 \\
Prefixo Cíclico & Normal \\
$\mathrm{N}^{\circ}$ de Antenas & 1 \\
MIMO & Não \\
Capacidade do Canal & $\sim 25 \mathrm{Mbps}$ \\
Taxa de Dados & $\sim 10 \mathrm{Mbps}$ \\
\hline
\end{tabular}

Tabela 5. Potência de Transmissão

\begin{tabular}{lll}
\hline \multicolumn{3}{c}{ Potência de Transmissão } \\
\hline BS (LTE-U) & $23 \mathrm{dBmW}$ & $\sim 200 \mathrm{~mW}$ \\
AP (Wi-Fi) & $17 \mathrm{dBmW}$ & $\sim 50 \mathrm{~mW}$ \\
\hline
\end{tabular}

Tabela 7. Parâmetros da UFMC

\begin{tabular}{ll}
\hline \multicolumn{2}{c}{ Parâmetros da UFMC } \\
\hline Atenuação Slobe & $20 \mathrm{~dB}$ \\
$\mathrm{~N}^{\circ}$ IFFT - $\mathrm{N}_{F T T}$ & 2048 \\
Tamanho da Subbanda & 12 \\
Número de Subbandas & 100 \\
\hline
\end{tabular}

Tabela 4. Parâmetros do Wi-Fi

\begin{tabular}{ll}
\hline \multicolumn{2}{c}{ Parâmetros Gerais do Wi-Fi } \\
\hline Frequência de Operação & $5 \mathrm{GHz}$ \\
Largura de Banda & $20 \mathrm{MHz}$ \\
Modulação & OFDM \\
Esquema de Modulação & QPSK \\
Padrão IEEE & $802.11 \mathrm{ac}$ \\
$\mathrm{N}^{\circ}$ Subportadoras & 52 \\
$\mathrm{~N}^{o}$ de Antenas do AP & 1 \\
Intervalo de Guarda & $800 \mathrm{~ns}$ \\
MIMO & $\mathrm{Não}$ \\
Capacidade do Canal & $14 \mathrm{Mbps}$ \\
Taxa de Dados & $\sim 7 \mathrm{Mbps}$ \\
\hline
\end{tabular}

Tabela 6. Parâmetros da F-OFDM

\begin{tabular}{ll}
\hline \multicolumn{2}{c}{ Parâmetros da F-OFDM } \\
\hline Tone Offset & 2.5 \\
Comprimento do Filtro & 1025 \\
\hline
\end{tabular}

Tabela 8. Parâmetros da FBMC

\begin{tabular}{ll}
\hline \multicolumn{2}{c}{ Parâmetros da FBMC } \\
\hline $\mathrm{N}^{\mathrm{o}}$ IFFT $-\mathrm{N}_{F T T}$ & 2048 \\
Tamanho do Filtro K & 4 \\
Número de Subportadoras & 1200 \\
\hline
\end{tabular}

A Tabelas 6, 7 e 8 mostram as configurações para as modulações F-OFDM, UFMC e FBMC, respectivamente. Alguns valores foram baseados nos diferentes trabalhos comparativos sobre as modulações candidatas citadas na introdução. Conforme a ITU-R [ITU 2012], os parâmetros de desvanecimento do canal do modelo Rayleigh Fading foram aplicados ao canal em que LTE-U e Wi-Fi estão coexistindo, como também adicionado o Add White Gaussian Noise - AWGN, que irão compor o cálculo do SINR.

\subsection{Avaliação Geral da Coexistência do Wi-Fi com LTE-U}

Os resultados do BER mostram que as modulações candidatas impactam na coexistência com o Wi-Fi no espectro não licenciado, haja vista os diferentes desempenhos dessas modulações sem a aplicação de técnica de coexistência, conforme as Figuras 5(a), 5(b) e 5(c). Por outro lado, percebe-se uma melhor vantagem ao tráfego Wi-Fi quando o LTE-U modula em F-OFDM e UFMC, mesmo modificando a quantidade de usuários, tornandose perceptível portanto, que tais modulações candidatas proporcionam diferentes desem- 
penhos, impactando no tráfego do Wi-Fi.

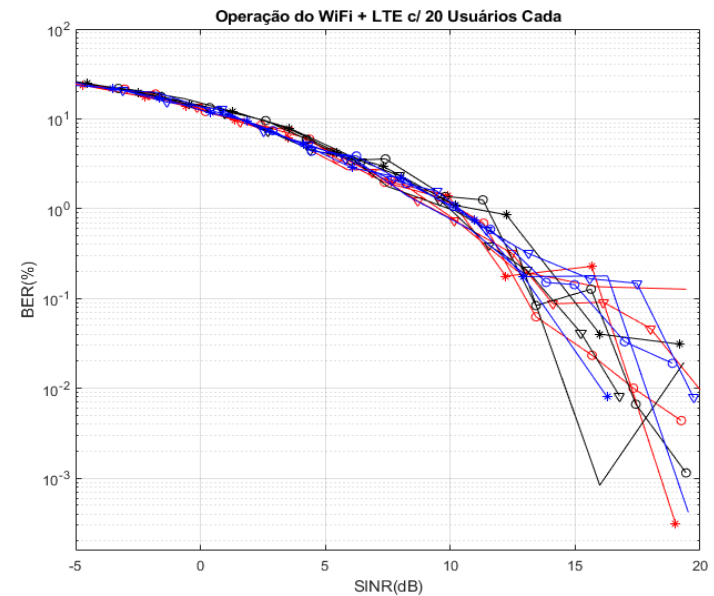

(a) BER x SINR 20 Usuários

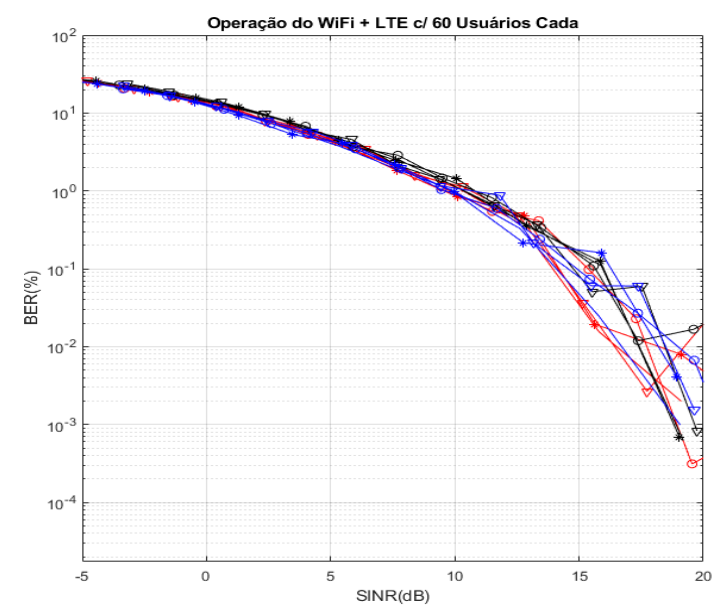

(c) BER x SINR 60 Usuários

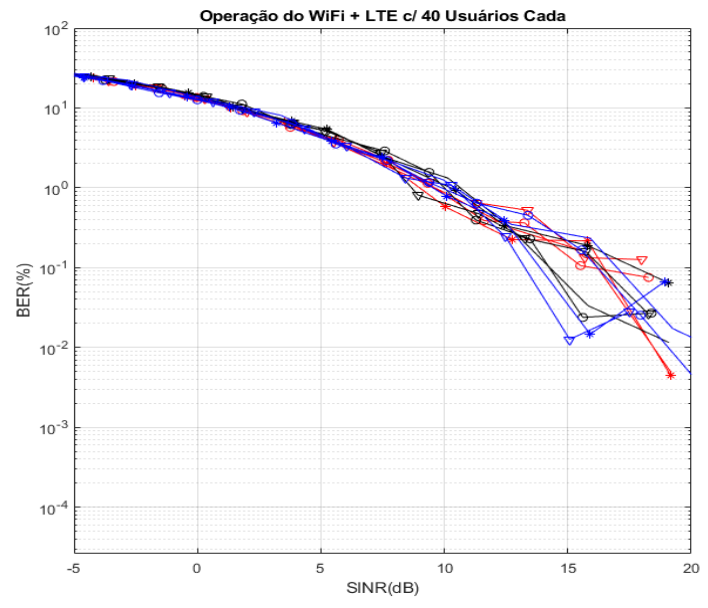

(b) BER x SINR 40 Usuários

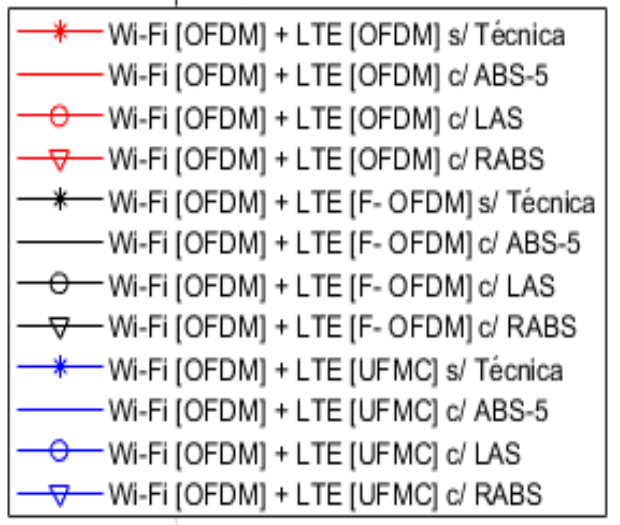

(d) Legenda

Figura 5. BER x SINR Nó Wi-Fi, do autor.

As Figuras 6(a), 6(b) e 6(c) mostram que, à medida que o cenário torna-se denso, as técnicas de coexistência começam a proporcionar melhor desempenho ao tráfego Wi$\mathrm{Fi}$, proporcionado pela combinação das técnicas propostas de coexistência LAS e RABS em comparação com a técnica ABS, quando combinadas com as modulações OFDM, F-OFDM e UFMC empregadas pelo LTE-U.

As Figuras 7(a), 7(b) e 7(c) mostram as vazões alcançadas por cada modulação, com e sem a combinação das técnicas de coexistência, que compatibilizam-se com o PER das Figuras 6(a), 6(b) e 6(c), respectivamente, confirmando que as técnicas propostas de coexistência LAS e RABS conseguem otimizar o tráfego Wi-Fi em comparação coma ABS.

Logo, é possível perceber que o tráfego Wi-Fi sofre tanto o impacto da modulação empregada no LTE-U quanto da combinação da técnica de coexistência. As modulações F-OFDM e UFMC em comparação à OFDM (emprego atual no LTE) conseguiram favorecer o tráfego Wi-Fi na maioria das variações de número de nós e nos parâmetros PER e Vazão, isso porque a F-OFDM utiliza filtro nas sub-bandas e a UFMC uma filtragem com 


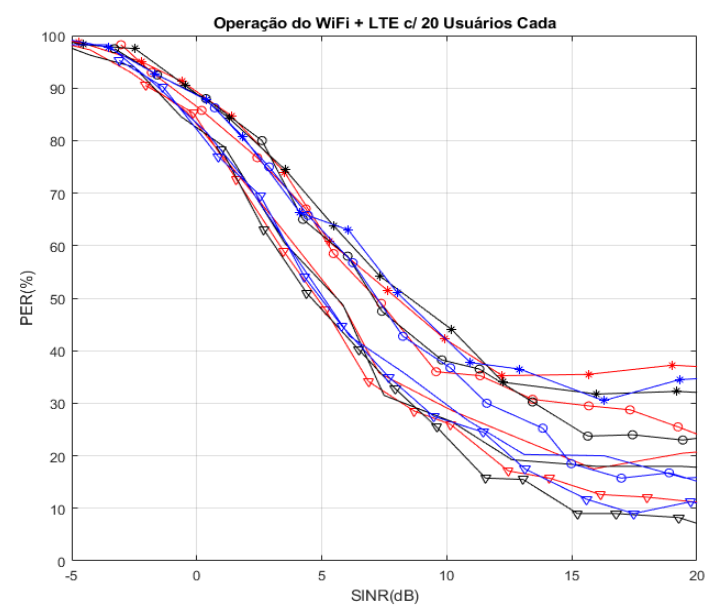

(a) PER x SINR 20 Usuários

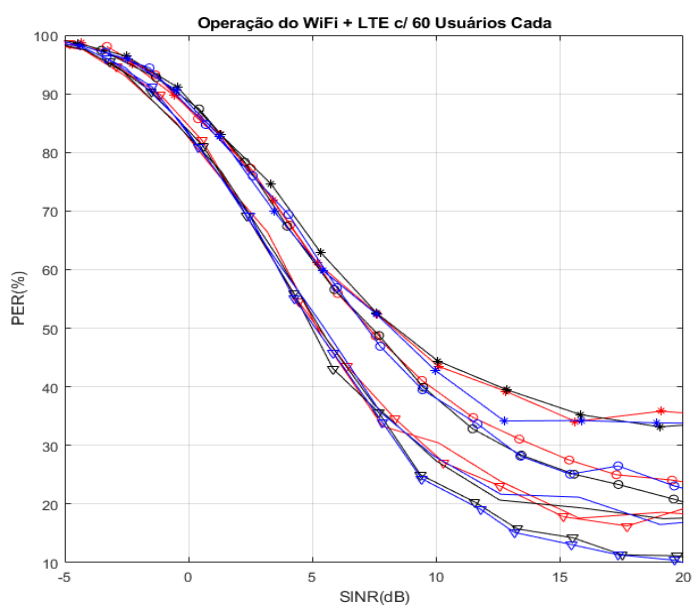

(c) PER x SINR 60 Usuários

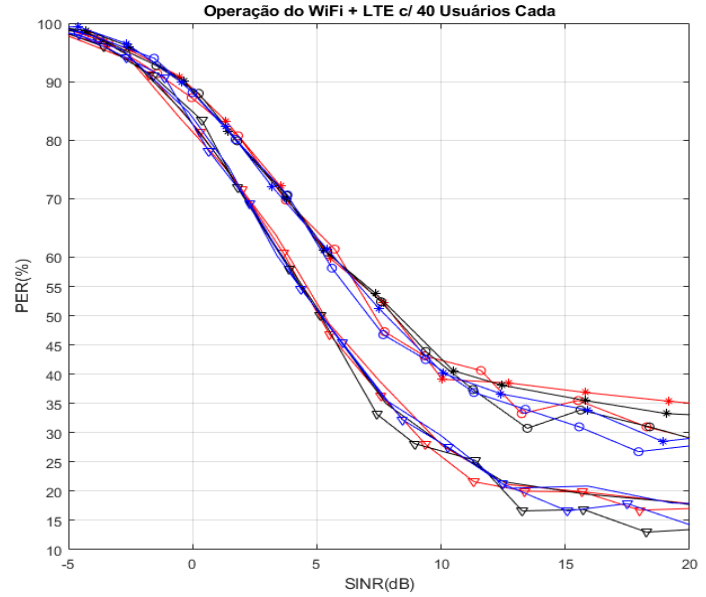

(b) PER x SINR 40 Usuários

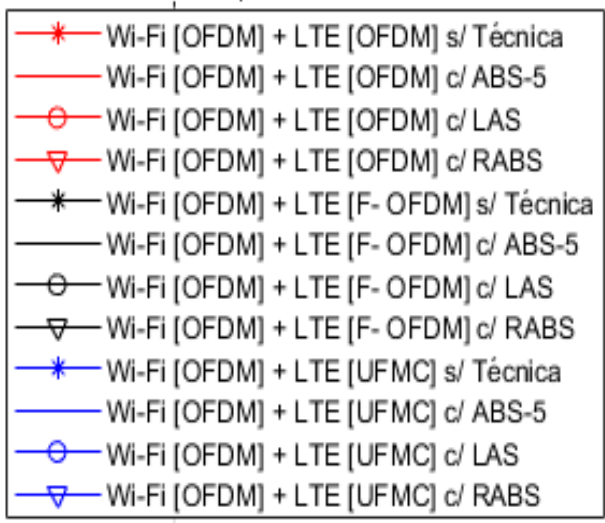

(d) Legenda

Figura 6. PER x SINR Nó Wi-Fi, do autor.

granularidade mais fina (sub-banda e subportadora) que resulta em baixa OoBE, menor ISI e boa eficiência espectral. Por outro lado, a técnica proposta RABS obteve os melhores índices de PER e Vazão quando empregadas com as modulações candidatas F-OFDM e UFMC.

\section{Conclusão e Trabalhos Futuros}

Esse trabalho propôs duas técnicas de coexistência para combinar às modulações do LTEU, no espectro não licenciado de $5 \mathrm{GHz}$, na operação simultânea entre o LTE-U e Wi-Fi, em um cenário denso, similar a small cell, onde utilizam aplicações para troca de mensagens em rede social online e/ou acesso a vídeos nas mais diversas plataformas de streaming, alterando-se o padrão de modulação do LTE-U de OFDM para F-OFDM e UFMC a cada simulação, bem como combinando-as com as técnicas de coexistência ABS, LAS e RABS.

Desse modo, é possível identificar a melhora do tráfego Wi-Fi ao combinar a técnica de coexistência RABS com as modulações F-OFDM e UFMC, empregadas no LTE-U. Com a operação da F-OFDM pelo LTE-U o tráfego Wi-Fi obteve as médias de 


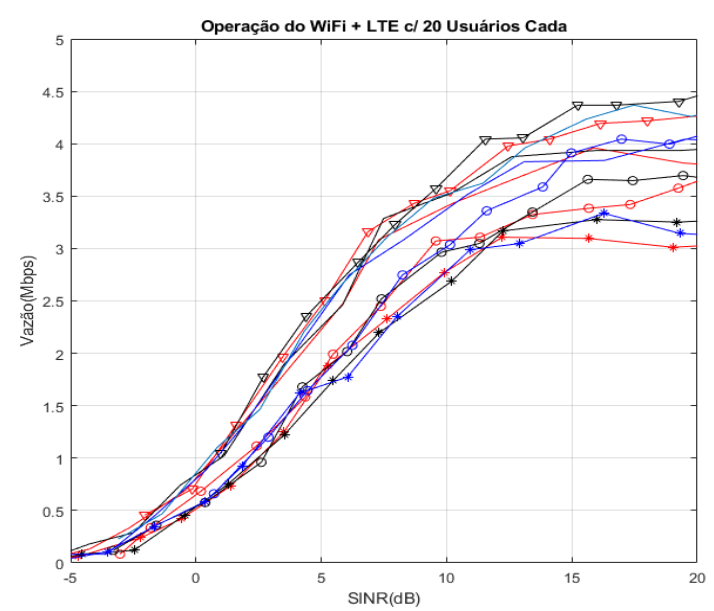

(a) Vazão x SINR 20 Usuários

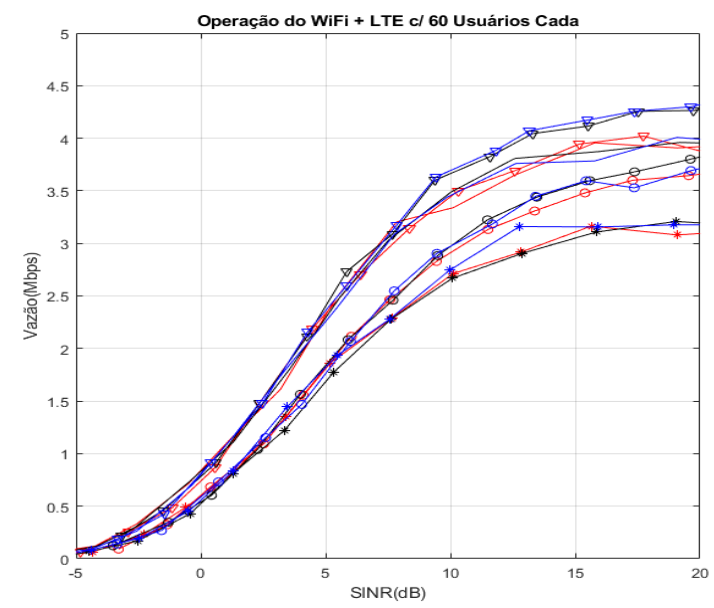

(c) Vazão x SINR 60 Usuários

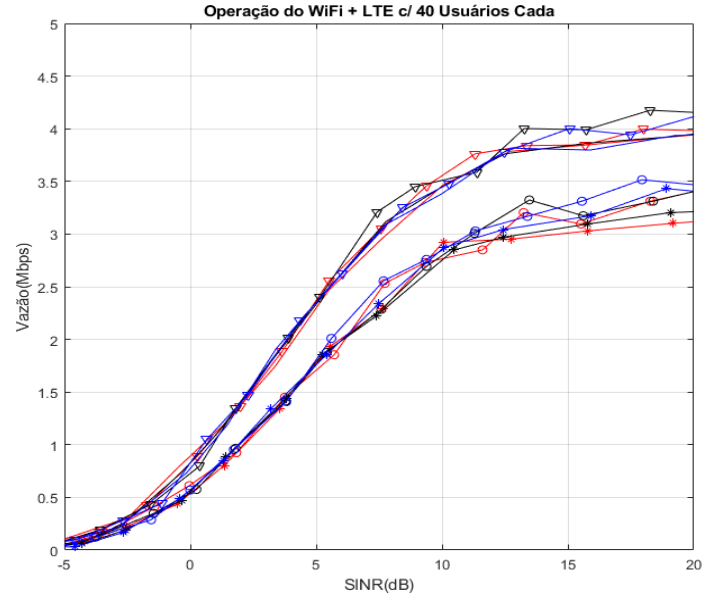

(b) Vazão x SINR 40 Usuários

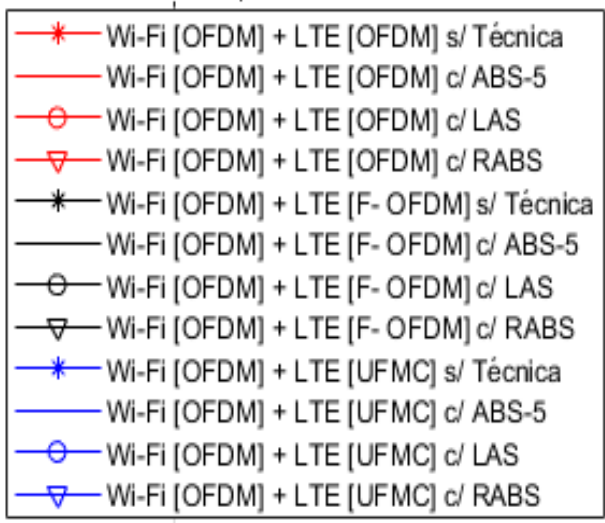

(d) Legenda

Figura 7. Vazão x SINR Nó Wi-Fi, do autor.

12\% de PER com 4,3Mbps de Vazão, com a UFMC as médias foram de 11,6\% de PER com 4,2Mbps de Vazão. Essas foram as melhores taxas de desempenho para o tráfego Wi-Fi na coexistência com o LTE-U.

Como trabalho futuro, pretendemos implementar um mecanismo de "escuta" do canal, similar ao da técnica LBT, a fim do LTE-U monitorar o canal de forma mais inteligente por meio de algoritmos de aprendizagem de máquina para que se possa adaptar a técnica RABS de forma mais dinâmica e inteligente conforme a intensidade do uso do canal pelo Wi-Fi.

\section{Referências}

3GPP (2009). Release 10 - 3rd generation partnership project (3gpp). http: / / www . 3gpp.org/specifications/releases/70-release-10.html. Acessado: 20 out. 2018.

3GPP (2016). Release 13 - 3rd generation partnership project (3gpp). http: / / www . 3gpp.org/release-13. Acessado: 13 jul. 2019. 
Abinader, F. M., de Sousa, V. A., Choudhury, S., Chaves, F. S., Cavalcante, A. M., Almeida, E. P. L., Vieira, R. D., Tuomaala, E., and Doppler, K. (2018). Lte/wi-fi coexistence in $5 \mathrm{ghz}$ ism spectrum: Issues, solutions and perspectives. Wireless Personal Communications, 99(1):403-430.

Beard, C. and Stallings, W. (2016). Wireless Communication Networks and Systems. Pearson.

Bocanegra, C., Kennouche, T. E., Li, Z., Favalli, L., Felice, M. D., and Chowdhury, K. (2019). E-fi: Evasive wi-fi measures for surviving lte within $5 \mathrm{ghz}$ unlicensed band. IEEE Transactions on Mobile Computing, 18(4):830-844.

Bonatelli, C. (2020). Redes 5g começam a chegar ao brasil, mas conexão ficará aquém do potencial. https:// link.estadao.com.br/noticias/cultura-digital, redes-5g-comecam-a-chegar-ao-brasil-mas-conexao-ficaral -aquem-do-potencial, 70003365488 . Acessado: 20 jul. 2020.

CISCO (2020). Cisco visual networking index: Global mobile data traffic forecast update, 2018-2023 white paper. Acessado: 13 abr. 2020.

Cui, H., Leung, V. C. M., Li, S., and Wang, X. (2017). Lte in the unlicensed band: Overview, challenges, and opportunities. IEEE Wireless Communications, 24(4):99105.

FCC (2016a). Part 15 - radio frequency devices. https://www.ecfr.gov/ cgi-bin/text-idx?SID=c79f7d57696072cbe1a68ca0870b1375\&mc= true\&node=pt 47.1.15\&rgn=div5. Acessado: 20 abr. 2020.

FCC (2016b). Special temporary authorization. https://apps.fcc.gov/els/ GetAtt.html?id=172096\&x. Acessado: 18 jul. 2019.

FCC (2018). Fcc test report. https: / / apps. fcc.gov/els/GetAtt.html ?id= $172096 \& \mathrm{x}$. Acessado: 18 jul. 2019.

Gerzaguet, R., Bartzoudis, N., Baltar, L. G., Berg, V., Doré, J.-B., Kténas, D., FontBach, O., Mestre, X., Payaró, M., Färber, M., and Roth, K. (2017). The 5g candidate waveform race: a comparison of complexity and performance. EURASIP Journal on Wireless Communications and Networking, 2017(1):13.

Huang, Y., Chen, Y., Hou, Y. T., Lou, W., and Reed, J. H. (2018). Recent advances of lte/wifi coexistence in unlicensed spectrum. IEEE Network, 32(2):107-113.

Ichkov, A., Atanasovski, V., and Gavrilovska, L. (2017). Potentials for application of millimeter wave communications in cellular networks. Wireless Personal Communications, 92(1):279-295.

ITU, I. T. U. (2012). Propagation data and prediction methods for the planning of indoor radiocommunication systems and radio local area networks in the frequency range 900 $\mathrm{mhz}$ to $100 \mathrm{ghz}$. Recomendations ITU-R, P Series(p.1238-7).

Liu, Y., Chen, X., Zhong, Z., Ai, B., Miao, D., Zhao, Z., Sun, J., Teng, Y., and Guan, H. (2016). Waveform candidates for 5g networks: Analysis and comparison. CoRR, abs/1609.02427. 
Lizeaga, A., Rodriguez, P., Val, I., and Mendicute, M. (2017). Evaluation of wcp-coqam, gfdm-oqam and fbmc-oqam for industrial wireless communications with cognitive radio. Journal of Computer Networks and Communications.

MULTEFIRE (2018). Multefire specification 1.0.1. https://www. multefire. org/mfa-v1-0-january-2018/. Acessado: 18 jul. 2019.

QUALCOMM (2014). Qualcomm research lte in unlicensed spectrum: Harmonious coexistence with wi-fi. https://www. qualcomm.com/media/documents/ files/lte-unlicensed-coexistence-whitepaper.pdf. Acessado: 18 jul. 2018.

Sun, H., Fang, Z., Liu, Q., Lu, Z., and Zhu, T. (2017). Enabling lte and wifi coexisting in $5 \mathrm{ghz}$ for efficient spectrum utilization. 2017:1-17.

Tariq, M., Anjum, M. R., and Amjad, M. (2018). Design of simulation system for lte-u using $5 \mathrm{ghz}$ band in matlab. Wireless Personal Communications, 100(4):1661-1676. 